\title{
Sensitivity, prediction uncertainty and detection limit for artificial neural network calibrations
}

Franco Allegrini and Alejandro C. Olivieri*

Departamento de Química Analítica, Facultad de Ciencias Bioquímicas y Farmacéuticas, Universidad Nacional de Rosario, Instituto de Química de Rosario (IQUIR-CONICET), Suipacha 531, Rosario S2002LRK, Argentina

* Corresponding author, fax +54-341-4372704, e-mail: olivieri@iquir-conicet.gov.ar 


\section{TABLE OF CONTENTS}

Monte Carlo simulations .......................................................

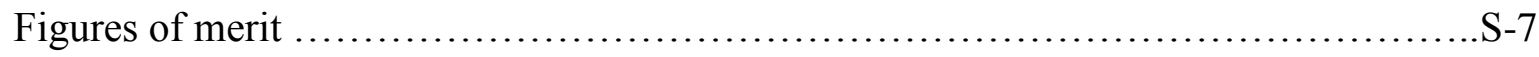

Plots of $\mathbf{b}_{\text {ANN vectors } \ldots \ldots \ldots \ldots \ldots \ldots \ldots \ldots \ldots \ldots \ldots \ldots \ldots \ldots \ldots \ldots \ldots \ldots \ldots \ldots \ldots \ldots \ldots \ldots \ldots \ldots \ldots}-8$

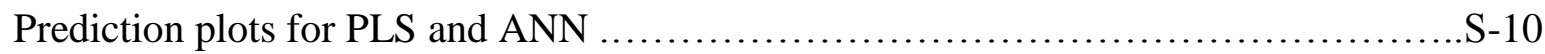

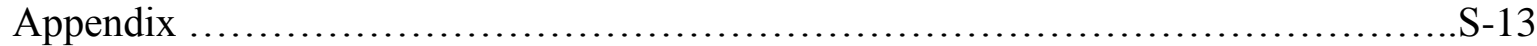

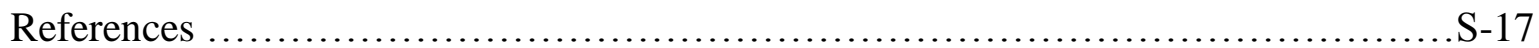




\section{Monte Carlo simulations}

One way to check that the equations for prediction uncertainty are correct is by means of noise addition Monte Carlo calculations. This involves adding a small amount of noise to test sample spectra, predicting the analyte concentrations and registering a statistics of the latter values. The computed standard deviation of the predicted values is plotted against the value of $\sigma_{y}$ given by equation (1) for iid noise or by equation (2) for other noise structures.

$$
\begin{aligned}
& \sigma_{\hat{y}}^{2}=\sigma_{x}^{2} \mathrm{SEN}^{-2}+h \sigma_{x}^{2} \mathrm{SEN}^{-2}+h \sigma_{\text {ycal }}^{2} \\
& \sigma_{\hat{y}}^{2}=\mathbf{b}_{\mathrm{ANN}}^{\mathrm{T}} \boldsymbol{\Sigma}_{x} \mathbf{b}_{\mathrm{ANN}}+h \mathbf{b}_{\mathrm{ANN}}^{\mathrm{T}} \boldsymbol{\Sigma}_{x} \mathbf{b}_{\mathrm{ANN}}+h \sigma_{\mathrm{ycal}}^{2}
\end{aligned}
$$

where the symbols are explained in the main text.

The results for iid noise addition were first studied for typical test samples of the three studied systems. A small amount of noise (see text for specific noise levels) was repeatedly added (10,000 times), using different random seeds each time, to a given test

spectrum, in the case where all sample components are present. The above calculations were repeated for a number of test samples of varying spectral overlap and concentrations. Noise was added in four different manners: (1) in the test signals only, (2) in calibration signals only, (3) in calibration concentrations only and (4) in all the three latter cases. This provides the opportunity to check the validity of each of the three terms of equation (1). The estimated value of $\sigma_{y}$ from the Monte Carlo approach can be compared with the theoretically predicted values using equation (1). This is graphically realized in Figure S1, where one can appreciate the agreement between Monte Carlo and theoretical values. 
Figures S2 and S3 show results analogous to those in Figure S1, in the case of the addition of pink and proportional noise respectively. The specific noise levels are detailed in the main manuscript text. It is apparent that good correlation is obtained between theoretical calculations and simulated results.

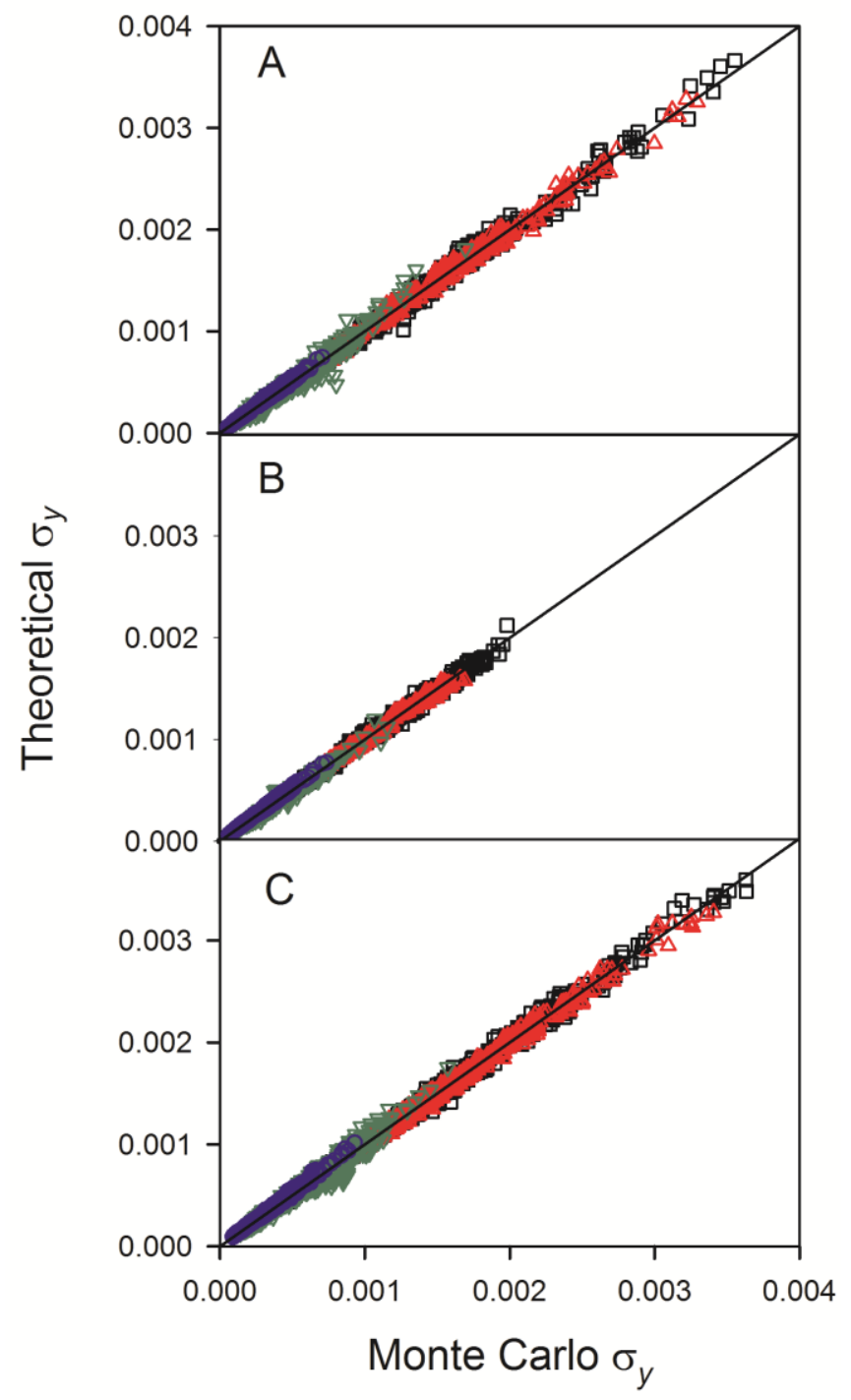

Figure S1. Theoretical prediction uncertainties as a function of Monte Carlo values, for 50 different test samples, 8 degrees of overlapping and 3 noise levels, in the case of iid noise addition (1,200 cases, see text for numerical details). Blue circles, noise only in calibration concentrations, green down triangles, noise only in calibration signals, red up triangles, noise only in test sample signals, black squares, noise in all concentrations and signals. The 
black solid lines indicate the perfect fit. A) Quadratic system. B) Sigmoidal system. C) Logarithmic system.

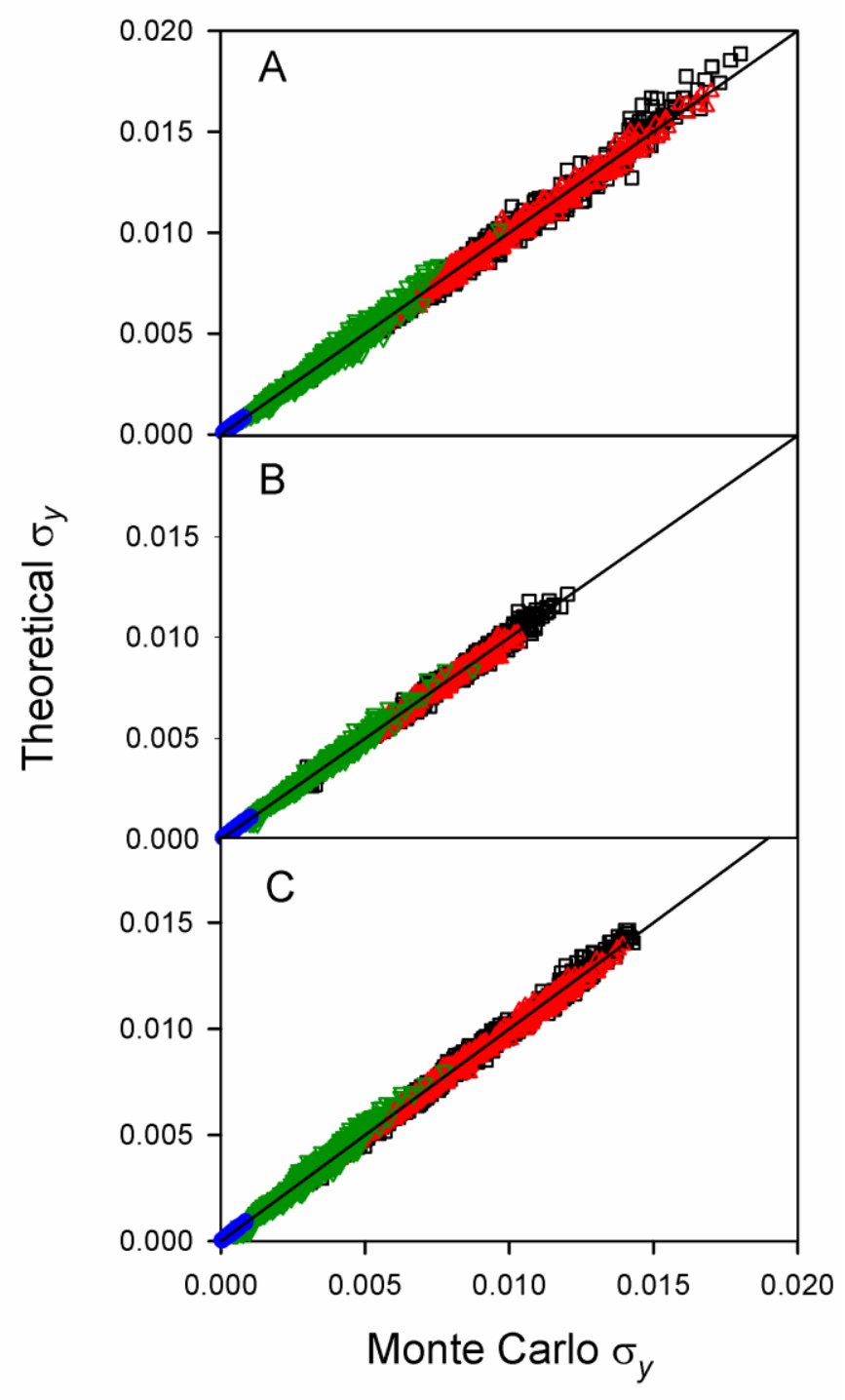

Figure S2. Theoretical prediction uncertainties as a function of Monte Carlo values, for 50 different test samples, 8 degrees of overlapping and 3 noise levels, in the case of pink noise addition (1,200 cases, see text for numerical details). Blue circles, noise only in calibration concentrations, green down triangles, noise only in calibration signals, red up triangles, noise only in test sample signals, black squares, noise in all concentrations and signals. The black solid lines indicate the perfect fit. A) Quadratic system. B) Sigmoidal system. C) Logarithmic system. 


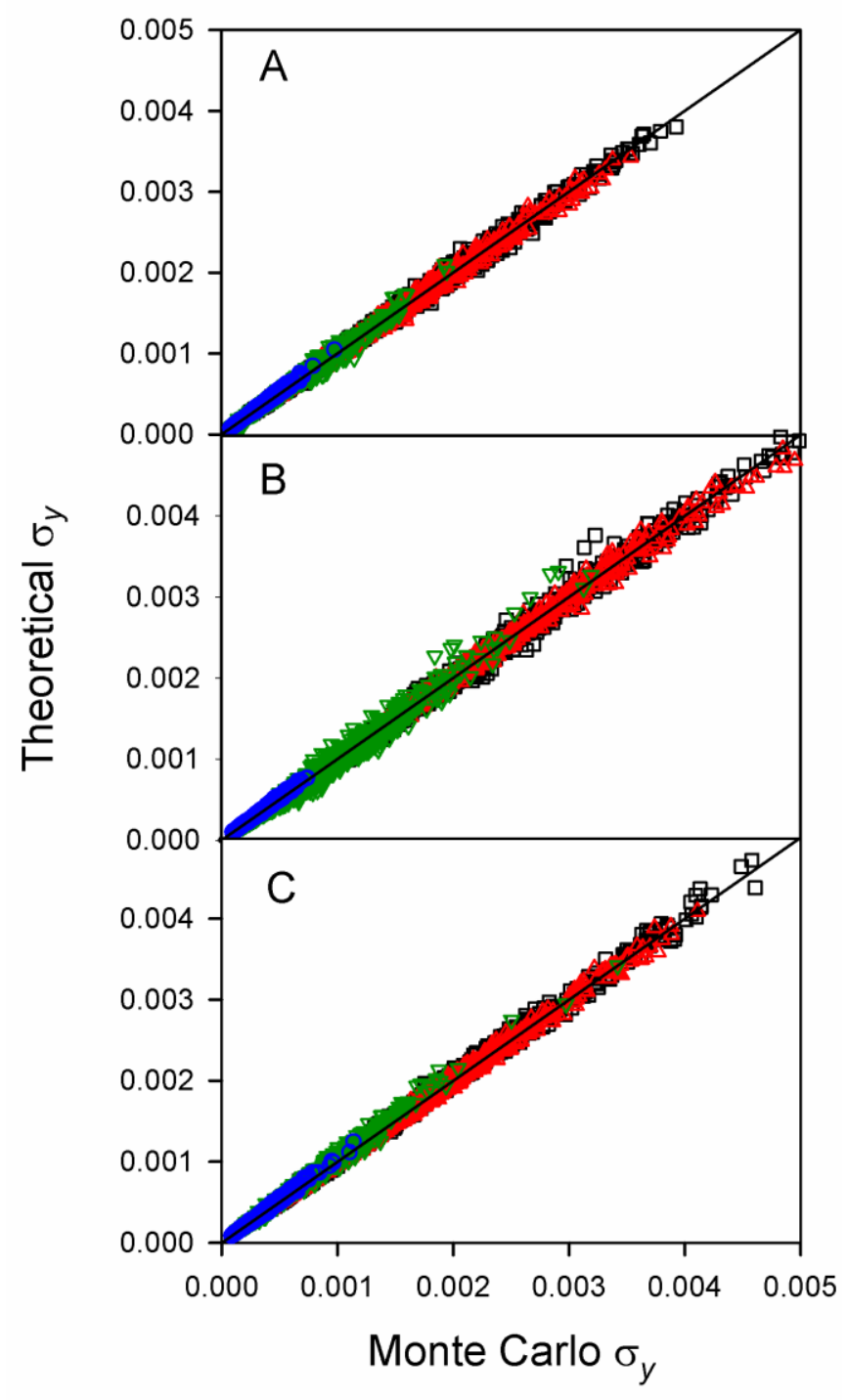

Figure S3. Theoretical prediction uncertainties as a function of Monte Carlo values, for 50 different test samples, 8 degrees of overlapping and 3 noise levels, in the case of proportional noise addition (1,200 cases, see text for numerical details). Blue circles, noise only in calibration concentrations, green down triangles, noise only in calibration signals, red up triangles, noise only in test sample signals, black squares, noise in all concentrations and signals. The black solid lines indicate the perfect fit. A) Quadratic system. B) Sigmoidal system. C) Logarithmic system. 


\section{Figures of merit}

Sensitivity and prediction uncertainty have been discussed in the main text. The Appendix gives MATLAB functions (ann_sen and ann_sen_sd) which provide the sensitivity and the prediction uncertainty for a set of test samples, given the calibration data and the calibration parameters.

To estimate the detection limit using an analogous approach to that previously reported for PLS regression, we start from the equation defining the hyper plane of analyte concentration equal to zero in the framework of RBF calibration. If data are mean centered so that the bias $w_{0}$ is not required, the predictive equation can be written as:

$$
y=\mathbf{w}^{\mathrm{T}} \mathbf{d}+\bar{y}_{\text {cal }}
$$

where $\mathbf{w}$ is the vector of weights and $\bar{y}_{\text {cal }}$ the mean calibration concentration. The latter is formally equivalent to that employed in PLS or PCR, where $\mathbf{w}$ takes the role of the vector of latent regression coefficients and $\mathbf{d}$ that of the test sample scores. However, in the linear regression methods PCR and PLS, the calibration scores are orthogonal to each other, a condition which is required to follow the derivation of ref. 1 . The RBF calibration design matrix $\mathbf{D}$ is not orthogonal, but it can be rotated to an orthogonal basis by means of singular value decomposition. Suitable projection onto the loadings of the latter decomposition of the design matrix $\mathbf{D}$, the sample design vectors $\mathbf{d}$ and the vector of weights $\mathbf{w}$ leads to a completely analogous formulation to that in ref. 1 . This means that the minimum and maximum limits of detection can be estimated by the following expressions:

$$
\begin{aligned}
& \mathrm{LOD}_{\min }=3.3\left(\mathbf{b}_{0 \mathrm{ANN}}^{\mathrm{T}} \Sigma_{x} \mathbf{b}_{0 \mathrm{ANN}}+h_{0 \min } \mathbf{b}_{0 \mathrm{ANN}}^{\mathrm{T}} \Sigma_{x} \mathbf{b}_{0 \mathrm{ANN}}+h_{0 \min } \sigma_{\text {ycal }}^{2}\right) \\
& \mathrm{LOD}_{\text {max }}=3.3\left(\mathbf{b}_{0 \mathrm{ANN}}^{\mathrm{T}} \Sigma_{x} \mathbf{b}_{0 \mathrm{ANN}}+h_{0 \text { mx }} \mathbf{b}_{0 \mathrm{ANN}}^{\mathrm{T}} \Sigma_{x} \mathbf{b}_{0 \mathrm{ANN}}+h_{0 \mathrm{max}} \sigma_{\text {ycal }}^{2}\right)
\end{aligned}
$$


where $\mathbf{b}_{\text {OANN }}$ is the $\mathbf{b}_{\mathrm{ANN}}$ vector corresponding to the minimum calibration concentration, which we assume close to the vector for a blank sample, and $h_{0 \min }$ and $h_{0 \max }$ are the minimum and maximum blank leverages. The latter ones are given by the following expressions:

$$
\begin{aligned}
& h_{0 \text { min }}=\frac{\bar{y}_{\text {cal }}^{2}}{\sum_{i=1}^{I} y_{i}^{2}}+\frac{1}{I} \\
& h_{0 \text { max }}=\max \left\{h_{\text {cal }}+h_{0 \min }\left[1-\left(\frac{y_{\text {cal }}}{\bar{y}_{\text {cal }}}\right)^{2}\right]\right\}+\frac{1}{I}
\end{aligned}
$$

where $h_{\text {cal }}$ and $y_{\text {cal }}$ are the leverage and (centered) analyte concentration of a generic calibration sample (see main text for the definition of $h_{\mathrm{cal}}$ ) and $I$ is the number of calibration samples. Additional details on the derivation of equations (6) and (7) can be found in ref. 1.

In the case of iid noise, equations (6) and (7) give simpler expressions:

$$
\begin{aligned}
& \mathrm{LOD}_{\min }=3.3\left(\sigma_{x}^{2} \mathrm{SEN}_{0}^{-2}+h_{0 \min } \sigma_{x}^{2} \mathrm{SEN}_{0}^{-2}+h_{0 \min } \sigma_{\text {ycal }}^{2}\right) \\
& \mathrm{LOD}_{\max }=3.3\left(\sigma_{x}^{2} \mathrm{SEN}_{0}^{-2}+h_{0 \max } \sigma_{x}^{2} \mathrm{SEN}_{0}^{-2}+h_{0 \max } \sigma_{\text {ycal }}^{2}\right)
\end{aligned}
$$

where $\mathrm{SEN}_{0}$ is the sensitivity for the calibration sample with minimum analyte concentration, taken to represent the value of this parameter for a blank sample.

The MATLAB function shown in the Appendix (ann_sen) permits estimation of the minimum and maximum detection limits.

\section{Plots of $b_{\text {ANN }}$ vectors}

For the simulated systems, the main text describes the training of appropriate ANNs based on RBF. Figure S4 compares the range spanned by all calibration $\mathbf{b}_{\mathrm{ANN}}$ vectors for 
the quadratic (Figure S4A), sigmoidal (Figure S4B) and logarithmic (Figure S4C) systems described in the main text, with the single vector of regression coefficients obtained by PLS calibration of the same data, for the largest degree of overlapping among sample components, i.e., the lowest selectivity for analyte 1 . As can be seen, the $\mathbf{b}_{\text {PLS }}$ vector, which is at the same time a predictor and SEN-estimator vector, lies within the boundaries of the range spanned by all $\mathbf{b}_{\mathrm{ANN}}$ vectors, which are only SEN-estimators but not predictors.

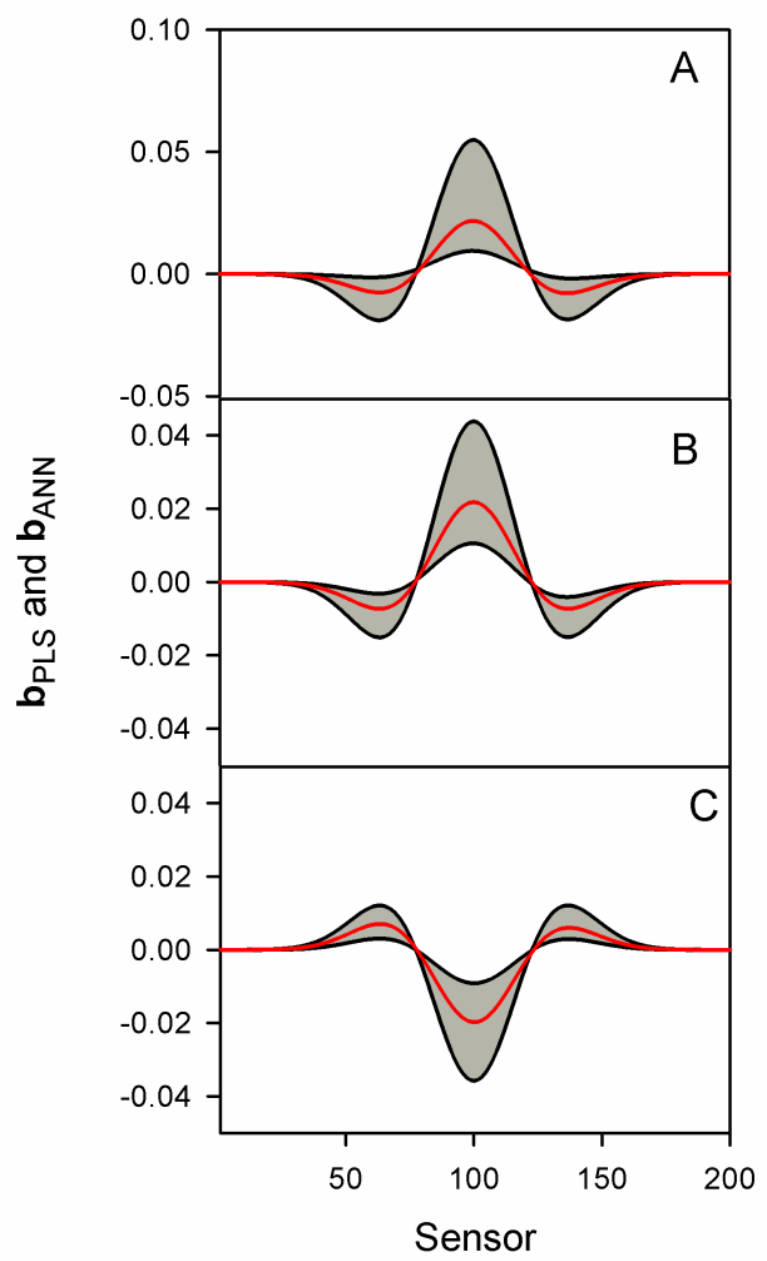

Figure S4. Plot of $\mathbf{b}_{\text {PLS }}$ (red), region spanned by $\mathbf{b}_{\text {ANN }}$ (gray). A) Quadratic system. B) Sigmoidal system. C) Logarithmic system. Results are shown for the spectral overlapping case of lowest selectivity towards analyte 1. 


\section{Prediction plots for PLS and ANN}

Figure S5 shows a plot of root mean square error for predicting the three properties in the experimental data set as a function of the number of principal components. The latter is equal to the number of input neurons in the ANN architecture. For each property, root mean square errors are shown for the calibration and test samples. The conclusion is that the selected number of principal components (15, see Table 2 in the main text) ensures stabilization of the average error by the trained neural networks.

Figure S6 shows predicted vs. nominal values for the test samples, in the case of the experimental data set, both for PLS and ANN models. For the three studied sample properties, it is apparent that calibration with the neural networks provides better analytical performance in comparison with the linear PLS models. These results are confirmed by the statistical analysis reported in Table 2 of the main text. 


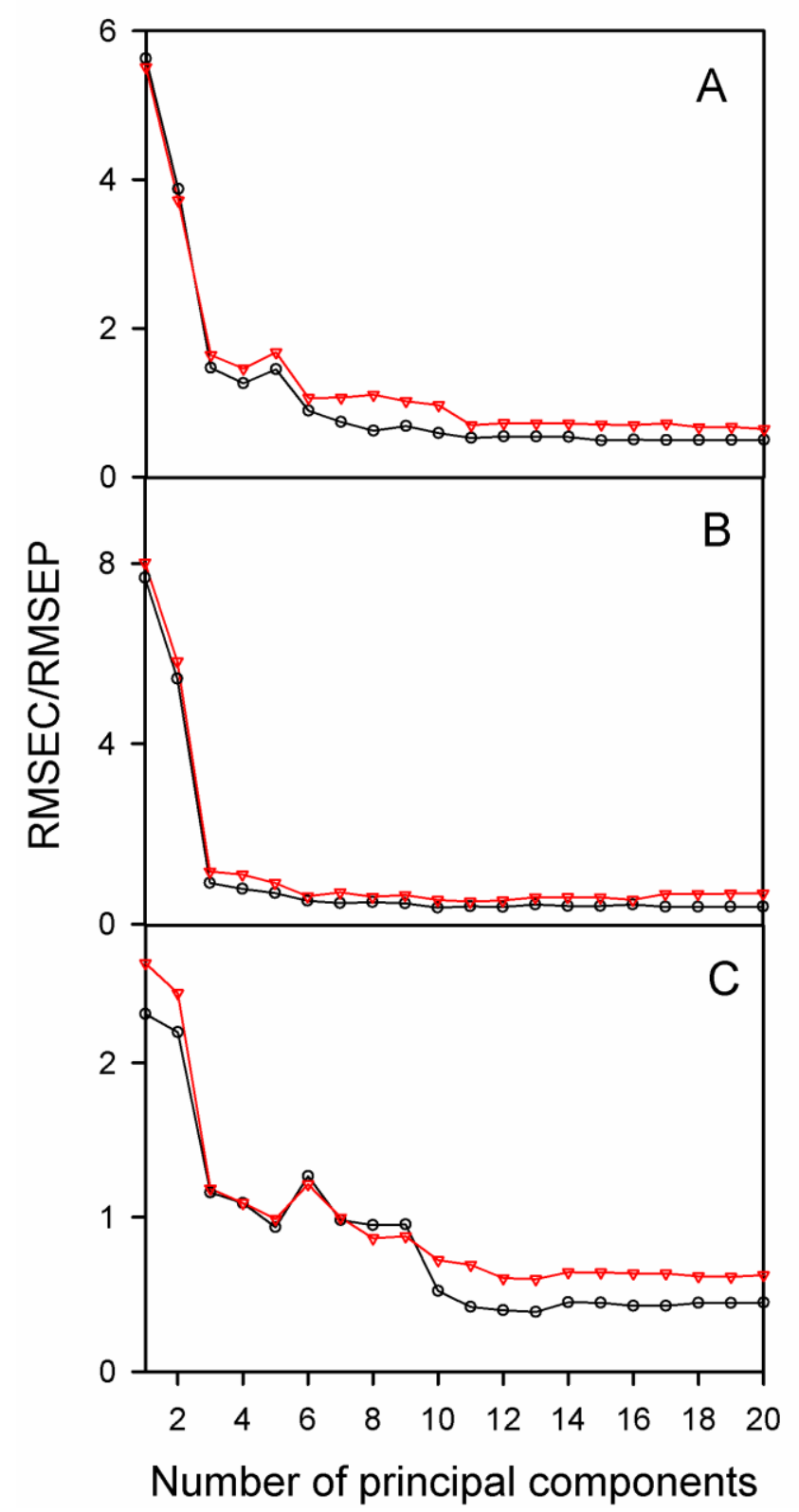

Figure S5. Root mean square error of calibration and prediction (RMSEC and RMSEP) as a function of the number of principal components used as input in the RBF calibration of the following properties in the experimental data set: A) fat, B) moisture, C) protein. Black circles, calibration RMSEC, red triangles, prediction RMSEP. 

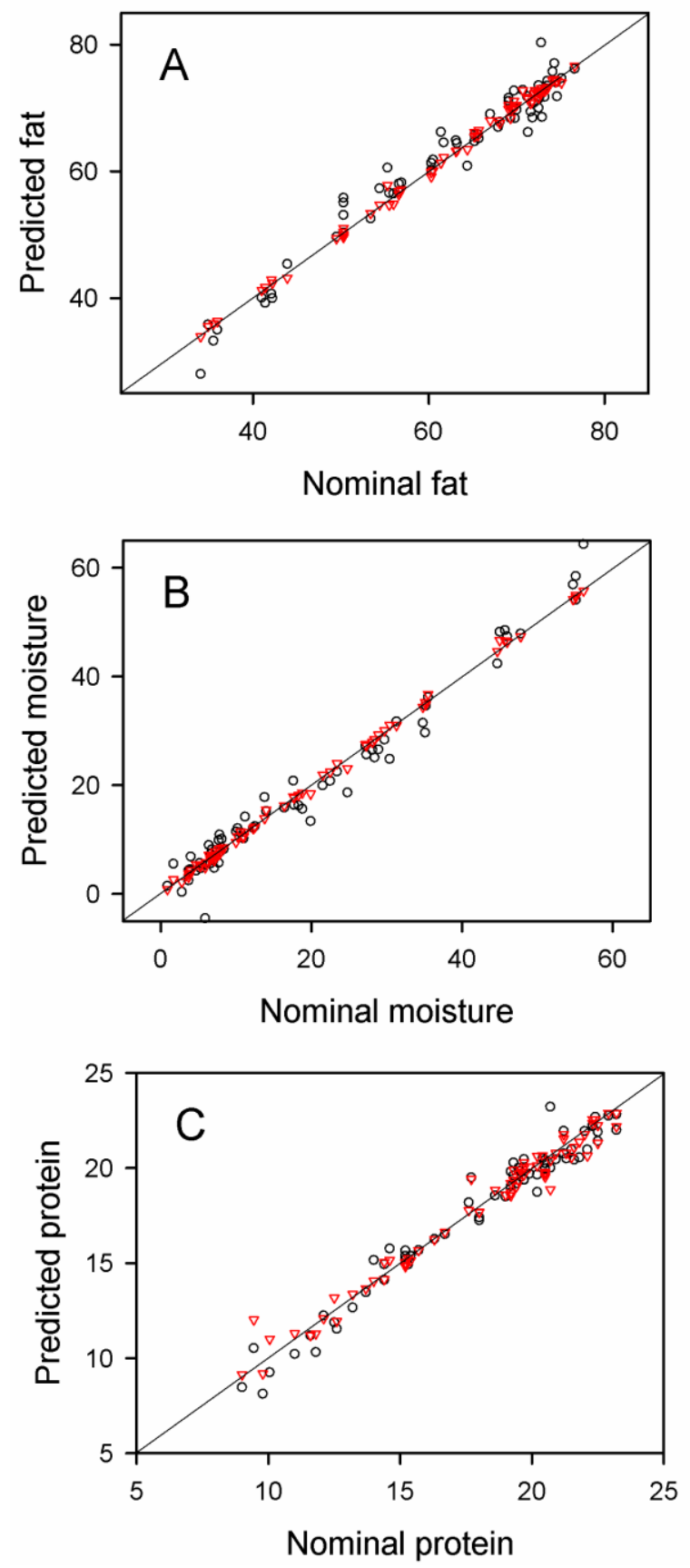

Figure S6. Predicted vs. nominal property values for the test samples in the experimental data set. Black circles correspond to predictions made by PLS, red triangles to RBF. A) Fat, B) moisture, C) protein determination. The thin solid line represents the ideal fit. 


\section{Appendix}

Two MATLAB codes are given as functions allowing to: (1) ann_sen, which estimates the sensitivity and detection limit for a calibration based on an RBF artificial neural network, and (2) ann_sen_sd, which provides the prediction uncertainty for a set of test samples. Both require access to the RBF MATLAB codes developed by Orr, available at www.anc.ed.ac.uk/mjo/software/rbf2.zip.

MATLAB function for sensitivity and detection limit:

function [lodmin,lodmax,avsen,minsen,maxsen]=ann_sen(X,y,Lv,sX,sy)

$\%$ INPUT

$\% \mathrm{X}=$ calibration data matrix (wavelenghts $\mathrm{x}$ samples)

$\%$ y = calibration concentrations vector (samples $x 1$ )

$\% L v=$ number of latent variables for PCA of $X$

$\% \mathrm{sx}=$ signal uncertainty

$\%$ sy = concentration uncertainty

$\%$

$\%$ OUTPUT

$\%$ lodmin = minimum detection limit

$\%$ lodmax = maximum detection limit

$\%$ avsen = average sensitivity

$\%$ minsen $=$ minimum sensitivity

$\%$ maxsen $=$ maximum sensitivity

\% Requires access to RBF program developed by Orr et al.

$\%$ see www.anc.ed.ac.uk/mjo/software/rbf2.zip

$\mathrm{I}=\operatorname{size}(\mathrm{X}, 2)$;

$\%$ Mean centering

meany=mean $(\mathrm{y})$;

$\mathrm{yc}=\mathrm{y}-\operatorname{mean}(\mathrm{y})$;

$\mathrm{Xc}=\mathrm{X}$-mean $(\mathrm{X}, 2)^{\star}$ ones $(1, \mathrm{I})$;

$\%$ PCA of $X$ 
$[\mathrm{U}, \mathrm{S}, \mathrm{V}]=\mathrm{svd}(\mathrm{Xc}, 0)$;

$\mathrm{TC}=\left(\mathrm{S}(1: \mathrm{Lv}, 1: \mathrm{LV})^{*} \mathrm{~V}(:, 1: \mathrm{Lv})^{\prime}\right)$;

$\%$ Calls to rbf code provided by Orr

$[C, R, w, i n f o, c o n f]=r b f \_f s \_2(T c, y c)$;

$D=r b f \_d m(T c, C, R)$;

$\%$ Sample leverages

for $i=1: I$

$$
h(i)=D(i,:)^{*} \operatorname{inv}\left(D^{\prime *} D\right)^{*} D(i,:)^{\prime} ;
$$

end

hmin=meany ${ }^{\wedge} 2 /$ norm $(y c)^{\wedge} 2+1 /$;

$h \max =\max \left(h^{\prime}+h \min { }^{*}\left(1-\left(y c .{ }^{\wedge} 2\right) /\right.\right.$ meany $\left.\left.^{\wedge} 2\right)\right)+1 / l$;

$\%$ Sensitivities

$[p, m]=\operatorname{size}(D)$;

$\mathrm{s}=\mathrm{zeros}(\operatorname{size}(\mathrm{X}))$;

$\mathrm{H}=\mathrm{zeros}(\mathrm{p}, \mathrm{m})$;

for samp $=1: p$

for $\mathrm{j}=1: \mathrm{m}$

$\mathrm{C}=\mathrm{C}(:, \mathrm{j})$;

$r=R(:, j)$;

$\mathrm{Z}=\operatorname{sum}\left(((\operatorname{Tc}(:, \operatorname{samp})-\mathrm{c}) . / \mathrm{r}) .{ }^{\wedge} 2\right)$;

$H($ samp,j) $=\exp (-z)$;

for $\mathrm{i}=1: \mathrm{Lv}$

$$
\mathrm{dH}(\mathrm{j}, \mathrm{i})=-\mathrm{w}(\mathrm{j})^{*} \mathrm{H}(\mathrm{samp}, \mathrm{j})^{\star} 2^{*}(\mathrm{Tc}(\mathrm{i}, \mathrm{samp})-\mathrm{c}(\mathrm{i})) / \mathrm{r}(\mathrm{i})^{\wedge} 2 ;
$$$$
s(:, s a m p)=s(:, s a m p)+d H(j, i)^{*} U(:, i) \text {; }
$$

end

end

$\operatorname{sen}(\operatorname{samp})=1 / \operatorname{norm}(s(:$, samp $))$;

end

$\%$ Output

$[a, b]=\min (y)$;

lodmin $=3.3^{*} \operatorname{sqrt}\left((1+h \min )^{\star} s x^{\wedge} 2 / \operatorname{sen}(b)^{\wedge} 2+s y^{\wedge} 2^{*} h \min \right)$; 
$\operatorname{lodmax}=3.3^{*} \operatorname{sqrt}\left((1+h \max )^{*} \operatorname{sx}{ }^{\wedge} 2 / \operatorname{sen}(b)^{\wedge} 2+s y^{\wedge} 2^{*} h \max \right)$;

avsen=mean(sen);

minsen $=\min (\operatorname{sen})$;

$\operatorname{maxsen}=\max ($ sen $)$;

MATLAB function for prediction uncertainty:

function [ypred,sd] $=a n n \_s e n \_s d(X, y, X t e s t, L v, s x, s y)$

$\%$ INPUT

$\% \mathrm{X}=$ calibration data matrix (wavelenghts $\mathrm{x}$ samples)

$\% \mathrm{y}=$ calibration concentrations vector (samples $\mathrm{x} 1$ )

$\%$ Xtest $=$ test data matrix (wavelenghts $\mathrm{x}$ samples)

$\% L v=$ number of latent variables for PCA of $X$

$\% \mathrm{sx}=$ signal uncertainty

$\%$ sy $=$ concentration uncertainty

$\%$

$\%$ OUTPUT

$\%$ ypred $=$ predicted analyte concentrations

$\% \mathrm{sd}=$ uncertainty in prediction

\% Requires access to RBF program developed by Orr et al.

$\%$ see www.anc.ed.ac.uk/mjo/software/rbf2.zip

$\mathrm{I}=\operatorname{size}(\mathrm{X}, 2)$;

$\mathrm{It}=\operatorname{size}(\mathrm{Xtest}, 2)$;

$\%$ Mean centering

meany $=$ mean $(\mathrm{y})$;

$\mathrm{yc}=\mathrm{y}-\mathrm{mean}(\mathrm{y})$;

$\mathrm{X} \mathrm{c}=\mathrm{X}$-mean $(\mathrm{X}, 2)^{*}$ ones $(1, \mathrm{I})$;

$\mathrm{Xtc}=$ Xtest-mean $(\mathrm{X}, 2)^{*}$ ones $(1, \mathrm{I})$;

$\%$ PCA of Xc

$[U, S, V]=s v d(X c, 0)$;

$\mathrm{TC}=\left(\mathrm{S}(1: \mathrm{Lv}, 1: \mathrm{Lv})^{*} \mathrm{~V}(:, 1: \mathrm{Lv})^{\prime}\right)$;

$\mathrm{Ttc}=\mathrm{U}(:, 1: \mathrm{Lv})^{\prime *} \mathrm{Xtc}$; 
$\%$ Calls to rbf code provided by Orr

$[\mathrm{C}, \mathrm{R}, \mathrm{w}$,info,conf] $]$ rbf_fs_2(Tc,yc);

$D=r b f \_d m(T c, C, R)$;

$\mathrm{Dt}=\mathrm{rbf} \_d \mathrm{dm}(\mathrm{Ttc}, \mathrm{C}, \mathrm{R})$;

ypred $=D t^{*} \mathrm{w}+$ meany;

$\%$ Sample leverages

for $i=1$ :It

$$
h(i)=\operatorname{Dt}(i,:)^{*} \operatorname{inv}\left(D^{\prime *} D\right)^{*} \operatorname{Dt}(i,:)^{\prime}+1 / l \text {; }
$$

end

$\%$ Sensitivities

$[p, m]=\operatorname{size}(D t)$;

$\mathrm{S}=\mathrm{zeros}(\operatorname{size}($ Xtest $))$;

$\mathrm{H}=\mathrm{zeros}(\mathrm{p}, \mathrm{m})$;

for samp $=1: p$

$$
\text { for } \mathrm{j}=1: \mathrm{m}
$$

$\mathrm{C}=\mathrm{C}(:, \mathrm{j})$;

$r=R(:, j)$;

$z=\operatorname{sum}\left(((\operatorname{Ttc}(:, \operatorname{samp})-c) . / r) \cdot{ }^{\wedge} 2\right) ;$

$H($ samp,j) $=\exp (-z)$;

for $i=1: L v$

$d H(j, i)=-w(j)^{*} H(\text { samp,j })^{*} 2^{*}(\operatorname{Ttc}(i, s a m p)-c(i)) / r(i) \wedge 2 ;$

$\mathrm{s}(:, \mathrm{samp})=\mathrm{s}(:, \mathrm{samp})+\mathrm{dH}(\mathrm{j}, \mathrm{i})^{\star} \mathrm{U}(:, \mathrm{i})$;

end

end

$\operatorname{sen}(\operatorname{samp})=1 /$ norm $(s(:$, samp $))$;

sd $($ samp,: $)=\operatorname{sqrt}\left((1+h(\operatorname{samp}))^{\star} \mathrm{sx}^{\wedge} 2 / \operatorname{sen}(\operatorname{samp})^{\wedge} 2+\mathrm{h}(\operatorname{samp})^{\star} \mathrm{sy}^{\wedge} 2\right)$;

end 


\section{References}

1 Allegrini, F. A.; Olivieri, A. C. Anal. Chem. 2014, 86, 7858-7866. 